\title{
Local protein synthesis in neuronal axons: why and how we study
}

\author{
Eunjin Kim \& Hosung Jung * \\ Department of Anatomy, Brain Research Institute, and Brain Korea 21 PLUS Project for Medical Science, Yonsei University College of \\ Medicine, Seoul 120-752, Korea
}

\begin{abstract}
Adaptive brain function and synaptic plasticity rely on dynamic regulation of local proteome. One way for the neuron to introduce new proteins to the axon terminal is to transport those from the cell body, which had long been thought as the only source of axonal proteins. Another way, which is the topic of this review, is synthesizing proteins on site by local mRNA translation. Recent evidence indicates that the axon stores a reservoir of translationally silent mRNAs and regulates their expression solely by translational control. Different stimuli to axons, such as guidance cues, growth factors, and nerve injury, promote translation of selective mRNAs, a process required for the axon's ability to respond to these cues. One of the critical questions in the field of axonal protein synthesis is how mRNA-specific local translation is regulated by extracellular cues. Here, we review current experimental techniques that can be used to answer this question. Furthermore, we discuss how new technologies can help us understand what biological processes are regulated by axonal protein synthesis in vivo. [BMB Reports 2015; 48(3): 139-146]
\end{abstract}

\section{INTRODUCTION}

A typical cell biology textbook published 20 years ago would state that all proteins in the axon of a vertebrate neuron come from the cell body. This 'tenet' of neuronal cell biology was based on thorough transmission electron microscopic observations of the sections from the adult rat brain, where the polyribosomes and rough endoplasmic reticulum were mainly found in neuronal cell bodies (1). In closer investigations, it was found that polyribosomes also localize to the base of den-

*Corresponding author. Tel: +82-2-2228-1651; Fax: +82-2-365-0700; E-mail: hosungjung@yonsei.ac.kr

http://dx.doi.org/10.5483/BMBRep.2015.48.3.010

Received 20 January 2015

Keywords: Axon, Axon-TRAP, BONCAT, Boyden chamber, Campenot chamber, Compartmentalized culture, Laser capture microdissection, Local mRNA translation, Microfluidic device, Neuron, Protein synthesis, Puromycin tagging, Ribosome, SILAC dritic spines (2). This finding immediately suggested a novel explanation to a long unanswered question in neuronal cell biology - how a new protein, synthesized in the cell body, is delivered to a specific postsynaptic site in highly complex dendritic arbors. The activated postsynapse can make the required protein on site by local mRNA translation (2). This idea led to a series of important discoveries that have firmly established local protein synthesis as one of key mechanisms underlying activity-dependent structural changes in the synapse (3). In contrast to the dendrite, however, polyribosomes were not readily detected on the other side of the synapse, the axon terminal. Moreover, most of the polyribosomes found in the axon localized to the axon initial segment, which is rather a 'postsynaptic' site for inhibitory synapses than a presynaptic structure (4). This observation led to a speculation that local protein synthesis may occur prevalently in postsynaptic sites, and evidence for protein synthetic machinery in axons in culture $(5,6)$ and in vivo $(7)$ did not draw much attention until the 1990 's, perhaps due to this rather strong notion. It was not until the early 2000's when two independent studies convincingly showed that axonal protein synthesis is required for specific biological processes such as chemotropic responses of growth cones (8) and potentiation of neurotransmitter release (9). These discoveries led to a rebirth of the field of axonal protein synthesis. Many follow-up studies have showed that diverse vertebrate neuronal axons are capable of local protein synthesis both during development and in adulthood and have identified several axonally localized mRNAs, whose local translation is required for axon guidance, elongation, survival, and regeneration (references in (10)).

The next goal in the field was to construct a comprehensive catalog of axonally localized mRNAs. Several genome-wide transcriptome analyses were performed aiming to gain insights into the roles that axonal protein synthesis may play, from the list of proteins that can be made from axonal transcriptome (11-15). However, unexpectedly large numbers of mRNAs were found in axons (sometimes thousands), which encode a variety of protein family members (even proteins that one would not normally expect there, such as transcription factors). Intriguingly, the contents of an axonal transcriptome is regulated by aging (15) and extrinsic cues (16), and only a select few of axonally localized mRNAs are translating at any given 
time (17). These results suggest that axons store mRNAs encoding proteins that are required in the near future and translate them only when required (Fig. 1).

At this time, conducting research on axonal protein synthesis often means asking one or all of the following questions: i) whether a particular protein is locally synthesized in the axon, ii) how the mRNA encoding that specific protein is chosen for translation from thousands of axonally localized mRNAs, and iii) what roles selective translation of that mRNA plays in the axon. Strategies to answer these questions differ depending on whether one has a particular protein of interest (candidatebased approach) or not (unbiased approach), but generally involve a combination of techniques in compartmentalized cell culture, molecular biology, biochemistry, and imaging. These methods can also be applied to study dendritic protein synthesis, but axons are more amenable for experiments as they are generally longer and therefore easier to isolate from their cell bodies.

\section{UNBIASED SCREENS FOR AXONALLY SYNTHESIZED PROTEINS}

The ultimate evidence that a particular protein is locally synthesized in the axon is that its abundance increases in mRNA translation-dependent manner in axons that are physically separated from their cell bodies. In unbiased screens, one selectively labels and/or purifies such proteins and identifies them by mass spectrometry (MS). Extracting proteins from axons with-

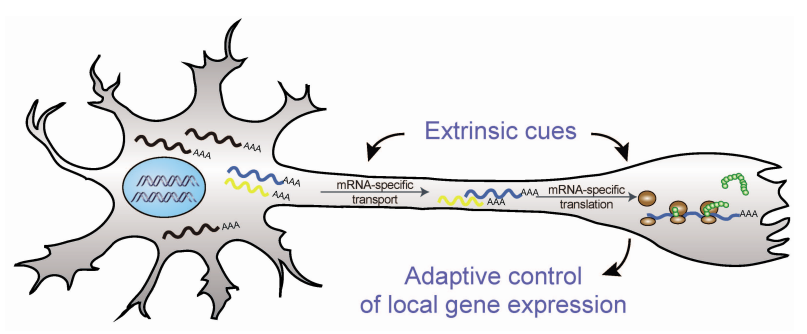

Fig. 1. Adaptive gene expression in the axon by local mRNA translation. out any trace of their cell bodies is critical, because slight contamination of somal proteins will lead to misidentification of axonally synthesized proteins. For this reason, all unbiased screens for axonally synthesized proteins published so far were conducted in cell culture systems, in which cell bodies can be experimentally eliminated from axons (which is often confirmed by absence of genomic DNA in axon lysate). For the same reason, proteins identified in unbiased screens must be validated by independent approaches, which are often imaging-based (see "Confirmation of axonal protein synthesis"). We start this section by introducing most common cell culture platforms used to separate axons from their cell bodies (Fig. 2 and Table 1).

\section{Preparation of 'axon-only' culture}

Campenot chamber: The Campenot chamber is the first device designed specifically for compartmentalized axon culture (18). It is a scaffold made of Teflon (a material known for non-stick coating of cooking pans), which is attached to a cell culture dish or cover glass via silicone grease (Fig. 2A). Currently,

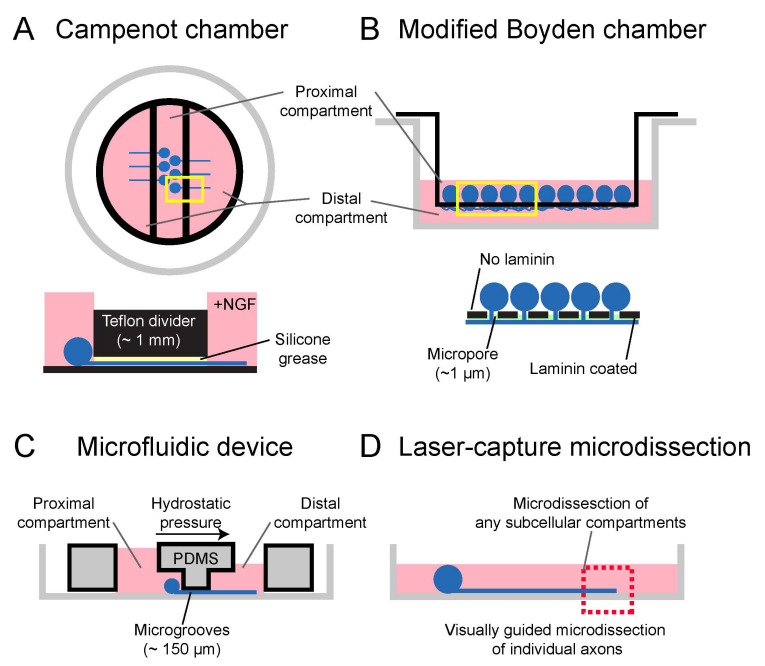

Fig. 2. Methods to collect pure axons.

Table 1. Comparison of compartmentalized axon culture platforms

\begin{tabular}{llllllll}
\hline \multicolumn{1}{c}{ Technique } & Consistency & Purity & Yield & Selectivity (1) & Fluidic separation & Explant culture? & Accessibility \\
\hline Campenot chamber & Normal & Good & Good & Good & Good & Yes & Good \\
Modified Boyden chamber & Good & Normal & Very good & Low & No & Yes & Very good \\
Microfluidic device & Good & Very good & Moderate & Very good & Very good & With modification & Good \\
Laser-capture microdissection & Very good & Excellent & Low & Excellent & No & Yes & Low \\
\hline
\end{tabular}

(1) Whether subcellular compartments within the axon can be selectively collected. Multi-chambered Campenot chambers and microfluidic device may be used to isolate proximal, middle and distal axons, separately Laser-capture microdissection allows selective isolation of minute structures such as the growth cone. 
there are many variations of the original three-chamber design, in which the dissociated neurons are plated in the central, or "proximal" compartment. Neuronal cell bodies and proximal neurites remain in the proximal chamber, and only the long neurites (which are likely to be axons) can pass through the silicone grease-culture dish interface under the Teflon divider into the "distal" compartments. With a complete seal, decent fluidic separation between compartments can be made, and axonal growth into the distal compartments is facilitated by selective addition of growth factors, such as nerve growth factor (NGF), to the "distal" compartments. The use of Campenot chambers involves relatively simple procedure (19) with commercially available resources. If a stable and uniform seal is made between the Teflon barrier and culture dish, it is possible to culture a pure population of axons with virtually no trace of cell bodies. In order to make a stable seal, however, the width of the barrier should be relatively large $(>1 \mathrm{~mm})$, which makes its use limited to neurons with very long axons. The making of a consistent seal requires training.

Modified Boyden chamber: The Boyden chamber was originally designed to study leukocyte migration, and it is made up of a porous cell culture insert that separates the cell culture dish into two compartments (20) (Fig. 2B). The size of pores can be modified so that only the axons can pass through them and into the distal compartment (approximately $1 \mu \mathrm{m}$ ), and the inserts with various pore sizes are commercially available. Neuronal cell bodies are plated on the upper surface of the insert, and axonal growth into the lower compartment is facilitated by selectively coating the lower surface of the insert with extracellular matrix (ECM) molecules (such as laminin) (21). The main advantage of this method is that the culture procedure is straightforward and that tissue explants (such as pieces of the brain and spinal cord) can be used without any modification. After axons grow into the lower "distal" compartment, all the cells in the upper "proximal" compartment can be removed by suction and scraping. With meticulous elimination, one can obtain pure axons with no detectable nuclear DNAs. Two main disadvantages of this method are that there is no fluidic separation between chambers (therefore cell bodies should be physically removed before any chemical stimulation, if axon-specific stimulation is required), and that it is difficult to get clear images of axons grown on a porous insert (plasma membrane can be stained with a fluorescent dye to improve image quality).

Microfluidic device: Microfluidic device was developed along the original idea of Campenot, but new material of transparent and biologically inert polymer (polydimethylsiloxane, or PDMS) was utilized (22). The PDMS device often contains two symmetrical chambers separated by narrow "microgrooves", through which only axons can grow. Gently pressing a dry PDMS mold on a dry coverslip or culture dish makes a watertight seal (Fig. 2C). Plating dissociated neurons in one chamber ("proximal" compartment) over a sufficient period of time allows axons to grow through the microgrooves into the other chamber ("distal" compartment). The main advantage of this technique is that almost perfect fluidic isolation between the two compartments can be achieved, simply by adjusting the volume of culture medium in each compartment. For example, when more culture medium is added to the proximal compartment, any chemical treated into the distal compartment will remain there because of the negative hydrostatic pressure in the microgrooves (Fig. 2C). PDMS molds with different sizes of microgrooves and chambers are commercially available or can be micro-fabricated. The microgrooves can be made significantly shorter than the barrier of the Campenot chamber (as short as $150 \mu \mathrm{m}$ ), and therefore, this method can be used for short axons and even for long dendrites. The transparency of PDMS makes it particularly suitable for live imaging. One disadvantage is that it is more expensive than other methods (unless you have access and expertise in micro-fabrication). Also, it takes some experience to make a good seal between a PDMS mold and ECM molecule-coated coverslip. Neurons that are plated not close enough to the microgrooves will not grow their axons into the distal compartment and therefore will be wasted. Plating the dissociated neurons close to the microgrooves also requires experience. Most commercially available PDMS molds may not be suitable for tissue explant culture, as cells have to be plated in a closed chamber that is covered by $\sim 100 \mu \mathrm{m}$-high roof.

Laser-capture microdissection: In laser-capture microdissection (LCM), one can dissect the selected regions from a tissue section or cell culture while directly observing the sample under the microscope (23) (Fig. 2D). To collect axons, individual axons are microdissected from neuron culture grown on an LCM-compatible slide. Neuron culture may be pre-stained with a fluorescent dye to aid visualization. This method provides the most precise way to collect axonal materials and enables subcellular compartment-specific microdissection of the axon (for example, axon shafts versus axon terminals) (15), which is not possible in any other compartmentalized cell culture systems. However, microdissection of individual axons means that one has to collect thousands of axons to obtain enough proteins or mRNAs for analysis. And it is sometimes necessary to fix the sample, because axons in culture are highly dynamic. LCM microscopes are generally expensive.

\section{Selective labeling and identification of axonally synthesized proteins}

In order to detect proteins that are locally synthesized in response to extrinsic cues, replicates of somaless axon culture are prepared by one of the methods described in the previous section, leaving one culture untreated and treating others with specific cues. Because severed axons can survive only for several hours in culture, any increase in protein abundance during cue stimulation may only be small and difficult to detect. It is particularly so, when an extrinsic cue promotes the synthesis of a protein that pre-existed in high abundance. Therefore, it is advantageous to analyze newly synthesized proteins, or the de 


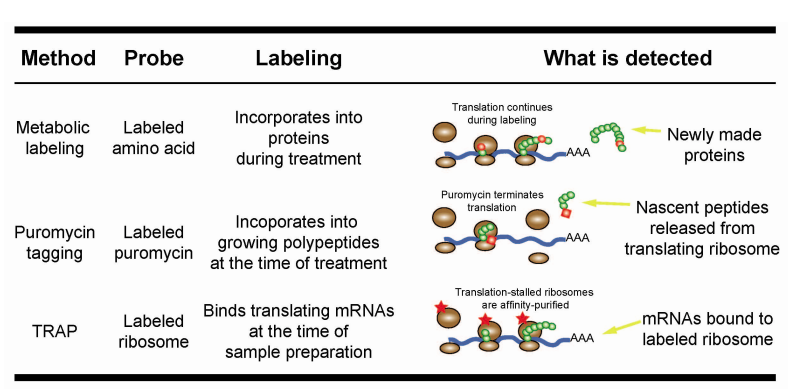

Fig. 3. Methods to identify axonally synthesized proteins.

novo proteome, rather than the steady-state proteome. Two standard biochemical approaches are available for selective labeling of de novo proteome: metabolic labeling of protein synthesis and puromycin tagging of nascent polypeptides (Fig. 3 and Table 2).

Metabolic labeling of protein synthesis: Metabolic labeling utilizes tagged amino acids, which live axons can use as building blocks for protein synthesis. Proteins synthesized after the addition of tagged amino acids are thus selectively labeled, which can be visualized, purified, and/or directly identified by MS (Fig. 3 and Table 2). Bioorthogonal noncanonical amino acid tagging (BONCAT) (24) and stable isotope labeling by amino acids in cell culture (SILAC) (25) are well-established techniques.

Tagged amino acids can be applied to severed axons (which can be cultured for several hours) or to intact axons cultured in a microfluidic device (or a well-prepared Campenot chamber). The latter method has advantages of avoiding the need to cut axons and being able to label axons for longer periods of time (over days). But, there is a risk that tagged amino acids may leak into the proximal compartment, which may label somal proteins that may be transported to axons. This will lead to false identification of axonally synthesized proteins and ruin the entire experiment. The former method can be done in diverse compartmentalized chambers, such as the Boyden chamber that gives a higher yield of axons, but severing axons may initiate unnecessary cellular responses.

BONCAT utilizes amino acid analogs, such as the Met ana$\log$ aziodohomoalanine (AHA), which contains an azide group. Axons are treated with $\mathrm{AHA}$, which is used for protein synthesis, resulting in labeling of de novo axonal proteome with the bioorthogonal azide group. These proteins are then covalently linked to an alkyne containing tag, such as fluorescent dyes or biotin, by Click chemistry (24). De novo axonal proteomes tagged to fluorescent dyes can be quantitatively analyzed by 2-dimensional differential gel electrophoresis (2D-DIGE) (17), and those tagged to biotin can be directly isolated by streptavidin affinity purification.

Once inside the axon, AHA is first charged to tRNA met by the enzyme Met-tRNA synthetase before being used for protein

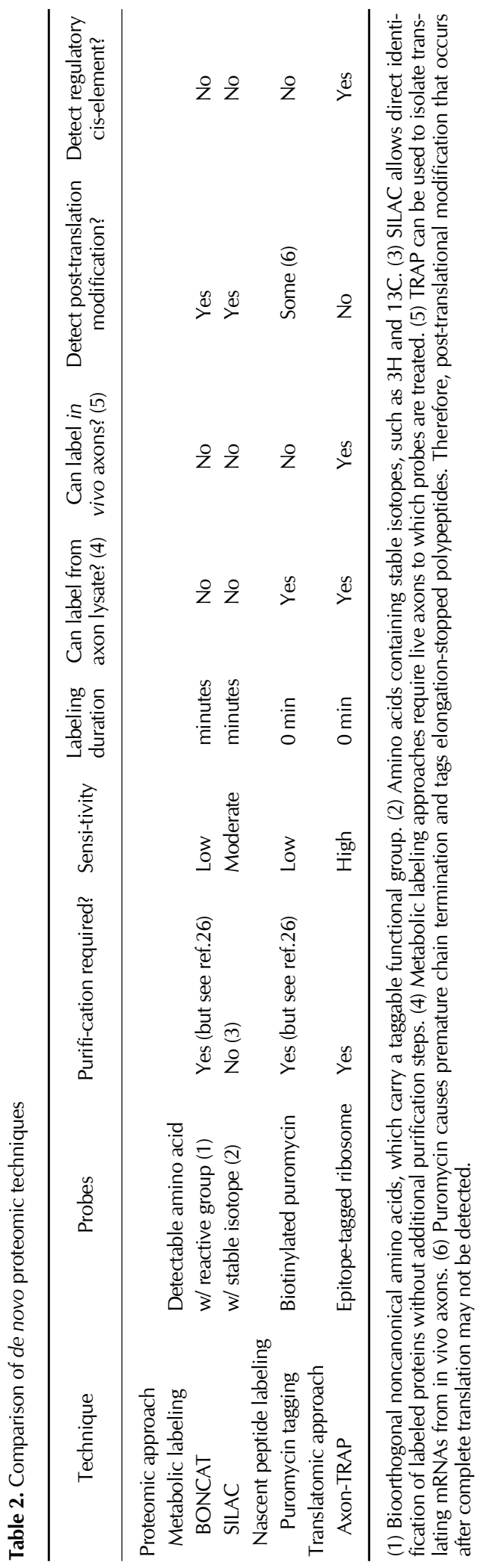

http://bmbreports.org 


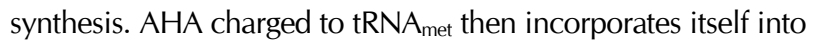
Met residues of nascent peptides during mRNA translation. The formation of AHA- $t R N A_{\text {met }}$ takes minutes, which means that there is always a lag between AHA treatment and actual proteome labeling. Axons should be cultured in Met- free culture medium to increase labeling efficiency, because AHA competes with Met for tRNA $\mathrm{Amt}_{\text {met }}$ and Met-tRNA synthetase.

SILAC utilizes amino acids containing stable isotopes (such as $3 \mathrm{H}, 13 \mathrm{C}$ or $15 \mathrm{~N}$ ), which can be used for protein synthesis. Similarly to BONCAT, SILAC requires time for the probe amino acids to be charged to appropriate tRNAs and specific amino acid-free medium (for example, Lys-free medium to use 13C-Lys) to increase labeling efficiency. Incorporation of the "heavy" amino acids (e.g. 13C-Lys) causes a predictable mass shift from the normal "light" amino acid (e.g. 12C-Lys). Heavy amino acid labeling causes no other chemical or functional changes to the labeled proteins, and therefore SILAC is noninvasive. By contrast, BONCAT may affect function of the protein it labels, because a noncanonical amino acid replaces an endogenous amino acid (e.g. AHA replaces Met). Another advantage of SILAC is that the labeled proteome can be directly identified by MS without any purification. Pre-existing proteome, which is "labeled" by the light isotope, can be distinguished by MS, and normalizing de novo proteome to steady state proteome enables quantitative analysis. In contrast, the BONCAT method normally requires purification of labeled proteome before their identification, which reduces the yield of protein recovery and produces biased enrichments of proteins. The recently developed technique for direct identification of biotinylated proteins by MS (26) may increase efficiency and accuracy of BONCAT-based analyses.

Puromycin labeling of nascent polypeptides: Puromycin tagging approach utilizes puromycin derivatives, which cause premature translation termination by incorporating themselves into the $\mathrm{C}$ terminus of nascent polypeptides. Therefore, this method differs from metabolic labeling approaches in the sense that it takes a snapshot of axonal protein synthesis at the time of puromycin treatment. Puromycin treated to axons lysate, as well as live axons, causes translation termination, and thus this approach does not require live axons. Instead, axon lysate can be obtained from intact neuron culture in the presence of emetine (which is an inhibitor of translation elongation, but unlike cycloheximide does not inhibit puromycin incorporation) (27). Biotinylated puromycin is added to axon lysate, labeling each translation-stalled, nascent polypeptide with a single puromycin tag at its carboxy terminus. These peptides are affinity-purified using streptavidin and identified by MS, but this may be combined with a recent technique to directly identify biotinylated proteins (26).

\section{Selective isolation of axonally translating mRNAs}

Most proteomic approaches utilize MS for protein identification. MS-based identification is less sensitive than DNAbased detection technologies, such as deep sequencing, main- ly because proteins cannot be amplified or fully sequenced. Therefore, although analyzing proteins is the most accurate way to study axonal protein synthesis, the use of nucleic acid-based technologies has its own merits. Nucleic acidbased approaches utilize the same principle of puromycin tagging. Information on de novo proteome is obtained from translation-stalled, ribosome-mRNA complexes. Instead of analyzing nascent polypeptides, however, this approach utilizes highly sensitive DNA-based technologies to get the sequence information of translating mRNAs. Ribosome-mRNA complexes can be purified either by the traditional polysome fractionation technique or ribosome immunoprecipitation. The particular strength of the latter approach, which is known as translating ribosome affinity purification (TRAP), is that a tagged ribosomal protein can be genetically encoded, enabling cell type-specific ribosome tagging (28).

Axon-TRAP: Like the puromycin tagging approach, TRAP takes a snapshot of axonal protein synthesis from axon lysate isolated from intact neurons. One advantage of this technique over biochemical approaches is that mRNAs can be identified by the RNA sequencing technology. This is not only more sensitive in identifying axonally synthesized proteins than MS, but it also has potential to identify regulatory cis-element in untranslated regions (UTRs). The second advantage, which may have a stronger impact, is that it can be used to study axonal translation in vivo. For example, the tagged ribosome can be selectively expressed in particular neurons, whose cell bodies are localized to one part of the brain (in vivo "proximal" compartment) and axons are localized to another part of the brain (in vivo "distal" compartment in culture). Although in vivo "distal" compartment may contain other cells in addition to the axons of interest, these cells do not express the tagged ribosome. Therefore, axonal ribosomes can be selectively retrieved from the lysate of the in vivo "distal" compartment, by affinity-purification. In this way, the mRNAs translating in axons of particular neurons can be isolated from an intact brain. The plausibility of this technique, axon-TRAP, has been confirmed recently, although it was used to measure translation of a few candidate mRNAs (17). It will be possible to perform RNA sequencing analysis of axon-TRAPed mRNAs in the future, which will reveal genome-wide information on the entire set of axonally translating mRNAs (translatome). One drawback of this technique is that ribosome immunoprecipitation does not distinguish ribosome-bound, translationally inactive mRNAs (which are often in RNA granules) from actively translating mRNAs. Combining axon-TRAP with ribosome run-off (29) or ribosome profiling (30) will enable us to perform a more accurate survey of axonal protein synthesis in vivo.

\section{CONFIRMATION OF AXONAL PROTEIN SYNTHESIS}

Regardless of whether one has found candidate mRNAs from unbiased screens or reasoning, their axonal synthesis must be experimentally validated. Because it is difficult to obtain 
enough axonal proteins for standard biochemical assays such as Western blotting, researchers often take a reasonable alternative, imaging based approaches, to measure local synthesis of a specific protein.

\section{Quantitative immunofluorescence (QIF)}

In QIF, the relative amount of a protein is measured by quantitative immunolabeling of fixed neurons, followed by quantitative fluorescent microscopy. Net immunofluorescence in the axon (i.e. fluorescent intensity in axon subtracted by background fluorescent intensity) represents the relative amount of the protein, which the antibody recognizes. To detect axonal protein synthesis, somaless axons are treated with an extrinsic cue or vehicle, and the abundance of a specific protein in axons is compared between groups. An increase in protein abundance, which can be blocked by translation inhibitors, indicates the axonal synthesis of that protein (8).

\section{Live imaging of translational reporter fluorescence}

It is well accepted that most cis-elements regulating translation of an mRNA reside in UTRs, particularly in the 3'-UTR. By expressing a chimeric mRNA consisting of the protein-coding sequence of a fluorescent protein and the UTRs of the gene of interest, translation of a particular mRNA can be visualized in real time. Membrane-targeted, destabilized fluorescent proteins are common reporter proteins, because they are not diffusible (therefore any fluorescence results from the local source) and are short-lived (therefore any fluorescence results from de novo protein synthesis) (31). Use of photoconvertible fluorescent de novo (such as Kaede and Dendra) (32) or fluorescent recovery after photobleaching (FRAP) (31) are some of the alternatives to measure translation by imaging.

\section{FUTURE DIRECTIONS}

Although axons contain hundreds or thousands of different mRNAs, inhibiting cue-induced translation of one or a few mRNAs almost completely blocks the axon's ability to respond to that cue (references in (33)), suggesting that cue-induced translation of selective mRNAs mediates specific biological processes. In this sense, an important question is how the different extrinsic cues activate translation of specific mRNAs. The first step in answering this question will be systematically analyzing extrinsic cue/target mRNA relationships. Axon-TRAP from axons treated with different cues would provide valuable information, which will lead to discoveries of new cis- and trans-acting elements that regulate message-specific translation in the axon.

Most of the unbiased screens for axonally synthesized proteins have been performed in cultured axons, mainly because it was impossible to selectively label or purify proteins from axons in vivo, which are normally intertwined with other cells. In this sense, the second important question is which proteins are synthesized in axons in vivo. Currently, axon-TRAP is the only technology that allows us to identify axonally synthesized proteins in vivo. Although translatome gives accurate information on de novo proteome, it does not tell us what happens after new proteins are made. For example, it has been suggested that axonally synthesized $\beta$-actin proteins differ from their pre-existing counterparts only by post-translational modification $(34,35)$, and this difference is key to newly made $\beta$-actin's ability to steer growing axons. Therefore, it will be important to develop new technologies that will enable direct and selective labeling of axonal proteome in vivo. A promising progress in this direction was recently made in Drosophila (36), and it will be exciting to see this technique applied to vertebrates.

Answering the first two questions will increase the number of mRNAs whose axonal translation may play key roles in vivo. For the clear understanding of their function, technologies need to be developed, which can selectively interfere with mRNA translation in axons, while leaving translation in the cell body intact. One promising strategy to decrease global translation activity selectively in the axon is chemically-inducible, genetically-encoded translational inhibitors $(37,38)$. Analogous to axon-TRAP, dormant inhibitor proteins could be expressed in specific neurons, whose cell bodies are localized to one part of the brain (in vivo "proximal" compartment). These inhibitor proteins could then be selectively activated in the axons of these neurons, by treating chemicals that induce inhibitor activity specifically to the other part of the brain, where their axon terminals are localized (in vivo "distal" compartment). To inhibit axonal translation of specific mRNAs, photoactivatable, caged antisense oligonucleotide (39) could be used, which can be delivered to specific neurons and uncaged selectively in the axon. An alternative approach is gene targeting of mRNA localization elements. For example, one can knock out the axon-specific splice variant, which contains a specific 3'-UTR, while leaving the entire protein coding region intact (40). This approach, however, requires the detailed knowledge on localization cis-elements of the target mRNA.

Recent development in DNA and protein detection technologies and new cell culture platform has made significant contribution to the field of axonal protein synthesis. Rapidly adopting and improving new technologies in diverse disciplines will be required to answer these remaining questions and pinpoint the biological significance of axonal protein synthesis in vivo.

\section{ACKNOWLEDGEMENTS}

We apologize for not being able to cite many important and relevant works due to space limitations. We thank T. Shigeoka for his valuable advice and critical reading of the manuscript. This work was supported by Basic Science Research Program (NRF-2013R1A1A1009625) and Bio \& Medical Technology Development Program (NRF-2014M3A9 B4043638) funded through NRF by the Korean government (MSIP) (HJ). 


\section{REFERENCES}

1. Peters A, Palay SL and Webster $d H$ (1971) Fine Structure of the Nervous System: Neurons and Their Supporting Cells, Oxford University Print.

2. Steward O and Levy WB (1982) Preferential localization of polyribosomes under the base of dendritic spines in granule cells of the dentate gyrus. J Neurosci 2, 284-291

3. Sutton MA and Schuman EM (2006) Dendritic protein synthesis, synaptic plasticity, and memory. Cell 127, 49-58

4. Steward O and Ribak CE (1986) Polyribosomes associated with synaptic specializations on axon initial segments: localization of protein-synthetic machinery at inhibitory synapses. J Neurosci 6, 3079-3085

5. Yamada KM, Spooner BS and Wessells NK (1971) Ultrastructure and function of growth cones and axons of cultured nerve cells. J Cell Biol 49, 614-635

6. Tennyson VM (1970) The fine structure of the axon and growth cone of the dorsal root neuroblast of the rabbit embryo. J Cell Biol 44, 62-79

7. Zelena J (1970) Ribosome-like particles in myelinated axons of the rat. Brain Res 24, 359-363

8. Campbell DS and Holt CE (2001) Chemotropic responses of retinal growth cones mediated by rapid local protein synthesis and degradation. Neuron 32, 1013-1026

9. Zhang X and Poo MM (2002) Localized synaptic potentiation by BDNF requires local protein synthesis in the developing axon. Neuron 36, 675-688

10. Jung $\mathrm{H}$, Yoon $B C$ and Holt $C E$ (2012) Axonal mRNA localization and local protein synthesis in nervous system assembly, maintenance and repair. Nat Rev Neurosci 13, 308-324

11. Minis A, Dahary D, Manor O, Leshkowitz D, Pilpel $Y$ and Yaron A (2014) Subcellular transcriptomics-dissection of the mRNA composition in the axonal compartment of sensory neurons. Dev Neurobiol 74, 365-381

12. Andreassi C, Zimmermann C, Mitter R et al (2010) An NGF-responsive element targets myo-inositol monophosphatase-1 mRNA to sympathetic neuron axons. Nat Neurosci 13, 291-301

13. Gumy LF, Yeo GS, Tung YC et al (2011) Transcriptome analysis of embryonic and adult sensory axons reveals changes in mRNA repertoire localization. RNA 17, 85-98

14. Taylor AM, Berchtold NC, Perreau VM, Tu CH, Li Jeon N and Cotman CW (2009) Axonal mRNA in uninjured and regenerating cortical mammalian axons. J Neurosci 29, 4697-4707

15. Zivraj KH, Tung YC, Piper M et al (2010) Subcellular profiling reveals distinct and developmentally regulated repertoire of growth cone mRNAs. J Neurosci 30, 15464-15478

16. Willis D, Li KW, Zheng JQ et al (2005) Differential transport and local translation of cytoskeletal, injury-response, and neurodegeneration protein mRNAs in axons. J Neurosci 25, 778-791

17. Yoon $B C$, Jung $\mathrm{H}$, Dwivedy $\mathrm{A}$, O'Hare $\mathrm{CM}$, Zivraj $\mathrm{KH}$ and Holt CE (2012) Local Translation of Extranuclear Lamin B Promotes Axon Maintenance. Cell 148, 1-13

18. Campenot RB (1977) Local control of neurite development by nerve growth factor. Proc Natl Acad Sci U S A
74, 4516-4519

19. Pazyra-Murphy MF and Segal RA (2008) Preparation and maintenance of dorsal root ganglia neurons in compartmented cultures. J Vis Exp 20, 951

20. Boyden S (1962) The chemotactic effect of mixtures of antibody and antigen on polymorphonuclear leucocytes. J Exp Med 115, 453-466

21. Willis DE and Twiss JL (2011) Profiling axonal mRNA transport. Methods Mol Biol 714, 335-352

22. Taylor AM, Blurton-Jones M, Rhee SW, Cribbs DH, Cotman CW and Jeon NL (2005) A microfluidic culture platform for CNS axonal injury, regeneration and transport. Nat Methods 2, 599-605

23. Emmert-Buck MR, Bonner RF, Smith PD et al (1996) Laser capture microdissection. Science 274, 998-1001

24. Dieterich DC, Link AJ, Graumann J, Tirrell DA and Schuman EM (2006) Selective identification of newly synthesized proteins in mammalian cells using bioorthogonal noncanonical amino acid tagging (BONCAT). Proc Natl Acad Sci U S A 103, 9482-9487

25. Ong SE, Blagoev B, Kratchmarova I et al (2002) Stable isotope labeling by amino acids in cell culture, SILAC, as a simple and accurate approach to expression proteomics. Mol Cell Proteomics 1, 376-386

26. Schiapparelli LM, McClatchy DB, Liu HH, Sharma $P$, Yates JR, 3rd and Cline HT (2014) Direct detection of biotinylated proteins by mass spectrometry. J Proteome Res 13, 3966-3978

27. Aviner R, Geiger T and Elroy-Stein O (2014) Genomewide identification and quantification of protein synthesis in cultured cells and whole tissues by puromycin- associated nascent chain proteomics (PUNCH-P). Nat Protoc 9, 751-760

28. Heiman M, Schaefer A, Gong S et al (2008) A translational profiling approach for the molecular characterization of CNS cell types. Cell 135, 738-748

29. Darnell JC, Van Driesche SJ, Zhang C et al (2011) FMRP stalls ribosomal translocation on mRNAs linked to synaptic function and autism. Cell 146, 247-261

30. Ingolia NT, Ghaemmaghami S, Newman JR and Weissman JS (2009) Genome-wide analysis in vivo of translation with nucleotide resolution using ribosome profiling. Science 324, 218-223

31. Aakalu G, Smith WB, Nguyen N, Jiang C and Schuman EM (2001) Dynamic visualization of local protein synthesis in hippocampal neurons. Neuron 30, 489-502

32. Leung KM and Holt CE (2008) Live visualization of protein synthesis in axonal growth cones by microinjection of photoconvertible Kaede into Xenopus embryos. Nat Protoc 3, 1318-1327

33. Jung $\mathrm{H}$ and Holt CE (2011) Local translation of mRNAs in neural development. Wiley Interdiscip Rev RNA 2, 153-165

34. Wang J, Boja ES, Tan W et al (2001) Reversible glutathionylation regulates actin polymerization in A431 cells. J Biol Chem 276, 47763-47766

35. Karakozova M, Kozak M, Wong CC et al (2006) Arginylation of beta-actin regulates actin cytoskeleton and cell motility. Science 313, 192-196

36. Elliott TS, Townsley FM, Bianco A et al (2014) Proteome 
labeling and protein identification in specific tissues and at specific developmental stages in an animal. Nat Biotechnol 32, 465-472

37. Je HS, Ji Y, Wang Y, Yang F, Wu W and Lu B (2011) Presynaptic protein synthesis required for NT-3-induced long-term synaptic modulation. Mol Brain 4, 1

38. Je HS, Lu Y, Yang F et al (2009) Chemically inducible inactivation of protein synthesis in genetically targeted neurons. J Neurosci 29, 6761-6766

39. Dmochowski IJ and Tang X (2007) Taking control of gene expression with light-activated oligonucleotides. Biotechniques $43,161,163,165$ passim

40. Perry RB, Doron-Mandel E, lavnilovitch E et al (2012) Subcellular knockout of importin beta1 perturbs axonal retrograde signaling. Neuron 75, 294-305 